\title{
BERNARD COVA
}

Euromed Management Marseille \& Università Bocconi Milan (Italie)

\section{PASCALE EZAN}

NIMEC - IAE de Rouen et Rouen Business School

\section{GREGORIO FUSCHILLO}

Euromed Management Marseille

\section{Zoom sur l'autoproduction du consommateur}

Les termes employés pour rendre compte de l'activité du consommateur sont aujourd'hui nombreux: coproduction, cocréation, collaboration, participation, etc. Cette profusion ne facilite pas la conceptualisation du rôle du consommateur ainsi que la qualification de ses actions. À l'inverse de ces approches globalisantes, nous proposons ici de faire un zoom sur l'autoproduction du consommateur. Pour ce faire, nous étudions des cas exemplaires permettant de générer deux nouvelles catégories d'autoproduction. La catégorisation ainsi obtenue souligne la nature polymorphe et dynamique du rôle actif du consommateur en relation avec l'entreprise. 


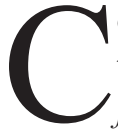
omme l'énonce l'appel à propositions pour ce dossier de la Revue française de gestion, "pour désigner le rôle actif du consommateur dans la vie des organisations, la littérature utilise une grande variété de termes. On parle de coproduction, de cocréation, de codesign, d'empowerment, de travail, etc., les définitions ne se recoupant pas forcément $»$. Sous la poussée de la nouvelle logique du marketing nommée Service-Dominant Logic (SDL) (Vargo et Lusch, 2004), un consensus a cependant semblé émerger durant la dernière décennie autour du terme de cocréation. Malheureusement, en voulant trop embrasser par un mouvement revendiqué de zoom arrière, c'est-à-dire par une analyse distanciée ne s'attachant qu'aux points essentiels communs à plusieurs phénomènes (Vargo et Lusch, 2011), les propagateurs de la notion de cocréation l'ont en partie vidée de son sens (Grönroos, 2011); toute interaction entre un consommateur et une entreprise pouvant être potentiellement considérée comme de la cocréation. Le même constat s'impose pour la notion de coproduction héritée du marketing des services et qui sert aujourd'hui d'ombrelle pour couvrir des phénomènes très différenciés.

Dans cet article, nous proposons d'opposer à ce mouvement englobant de zoom arrière, un zoom avant qui, selon Moss Kanter (2011), consiste à examiner plus en détail et de manière plus précise un phénomène. Il ne s'agit pas de couvrir l'ensemble du phénomène de brouillage des frontières entre les mondes de la consommation et de la production, mais, au contraire, de porter la focale sur une partie spécifique de ce phénomène. En écartant du champ de l'analyse tout ce qui relève du marketing participatif (Bonnemaizon et al.,
2012) ou consumer made (Cova, 2008), nous faisons un zoom sur l'activité que le consommateur développe, en relation plus ou moins étroite avec l'entreprise, afin de satisfaire ses besoins ou ceux de ses proches, et non ceux de l'entreprise. Cette activité d'un individu ayant comme objectif premier la production de biens et des services, pour sa propre consommation et celle de son entourage est nommée autoproduction (Pinçon, 1986). En nous concentrant sur le seul phénomène d'autoproduction, nous cherchons à le détailler de manière à en faire émerger toutes les nuances et subtilités. Troye et Supphellen (2012) positionnent l'autoproduction (self production en anglais) comme « un type spécifique de coproduction [...] dans laquelle les consommateurs utilisent des éléments préfabriqués par la marque pour produire quelque chose. Ceci est typique de nombreuses activités de consommation (cuisiner, jardiner, monter, maintenir) qui requièrent ou permettent différents niveaux d'effort productif » (Troye et Supphellen, 2012, p. 33-34). L'autoproduction du consommateur en relation avec l'entreprise est donc un concept qui ne vient pas s'opposer à la coproduction ou à la cocréation mais qui permet d'éclairer une partie précise du vaste phénomène de brouillage entre production et consommation.

L'autoproduction du consommateur en relation avec l'entreprise est déjà déclinée sous deux formes : l'autoproduction dirigée (Dujarier, 2008) et l'autoproduction accompagnée (Cerezuelle et Roustang, 2010). Posant le constat que ces formes prédéfinies ne prennent pas en compte les multiples facettes de l'autoproduction aujourd'hui (Montéty, 2010), ce travail vise à alimenter la réflexion dans ce domaine en construisant une catégorisation élargie du phénomène. 
Ce faisant, il contribue à (re)construire le socle de connaissances sur le rôle actif des consommateurs avec/dans les entreprises en évitant le centrage sur un seul acteur - le consommateur et son vécu - et, en offrant une vue systémique du jeu des acteurs de l'autoproduction et de leurs interactions : l'entreprise, le consommateur, et aussi, la communauté de consommateurs.

\section{I - DES DIFFICULTÉS POUR DÉCRIRE UNE RÉALITÉ NOUVELLE}

La profusion de propositions concernant l'activité productive du consommateur est tout à fait normale et renvoie au besoin de trouver les mots pour dire notre temps. En effet, en l'absence de concepts établis capables de décrire la réalité en mouvement, « il faut savoir se contenter des métaphores, des analogies, des images, toutes choses vaporeuses, qui seraient les moyens les moins mauvais possible pour dire "ce qui est", ce qui est à l'état naissant » (Maffesoli, 2000, p. IV). Ainsi, nombre de notions proposées pour dénommer le brouillage de la frontière producteur/ consommateur ne sont pas des concepts précis et réifiés mais plutôt des images provisoires, mouvantes et labiles, permettant d'accompagner le mouvement. Certains insistent cependant sur le fait que, bien que différents, ces termes participent d'une même mouvance qui mérite d'être comprise comme un tout : " Les termes de cocréation, coproduction et prosumption référent tous à des situations dans lesquelles les consommateurs collaborent avec les entreprises et d'autres consommateurs pour produire de la valeur. » (Humphreys et Grayson, 2008, p. 963). D'autres tentent de créer des liens entre ces termes (Xie et al., 2008).
Alors qu'en sociologie de la consommation, le néologisme « prosumption » (production/ consumption) est le plus utilisé aujourd'hui pour désigner l'activité des consommateurs (Ritzer et Jurgenson, 2010), la recherche en marketing et sur les consommateurs mobilise essentiellement deux termes : cocréation et coproduction. Le terme cocréation est plus particulièrement prôné par le courant de la SDL, Service-Dominant Logic (Vargo et Lusch, 2004), pour permettre un « zoom arrière » (Vargo et Lusch, 2011) sur le fonctionnement actuel des marchés. Le courant de la CCT, Consumer Culture Theory (Arnould et Thompson, 2005), fait du rôle cocréatif des consommateurs un pilier de sa théorie (Schau et al., 2009) et voit les consommateurs comme des agents interprétatifs capables de coproduction de sens et d'expériences (Arnould, 2007) par l'intégration des ressources des entreprises avec leurs propres ressources (Epp et Price, 2011). Le terme coproduction hérité du marketing des services (Eiglier et Langeard, 1987) est aussi repris par le courant de la SDL pour désigner la participation des acteurs au moment précis de la production de l'offre de services.

Malheureusement, ce semblant d'unanimité autour de l'articulation de la cocréation et et de la coproduction est contrarié par la confusion existant entre ces deux notions (tableau 1); Payne et al. (2008, p. 84) déclarant «ne faire aucune distinction entre ces deux termes ». Il est aussi battu en brèche par la pluralité des interprétations, parfois même contradictoires, de ces deux notions fonctionnant comme des ombrelles pouvant couvrir des réalités très différentes : S'agit-il de coproduction/ cocréation de valeur pour le consommateur organisée autour de son expérience et cen- 
trée sur ses besoins (Vargo et Lusch, 2008) ou s'agit-il d'une coproduction/cocréation de valeur pour l'entreprise (Humphreys et Grayson, 2008) organisée autour de son activité et centrée sur son offre ? Cette coproduction/cocréation est-elle encadrée (Marion, 2013) par l'entreprise ou spontanément développée par les consommateurs (Muniz et Schau, 2007) ? Pour reprendre une perspective expérientielle propre à la CCT (Carù et Cova, 2007), est-ce une expérience structurée par l'entreprise ou par le consommateur? Cette coproduction/ cocréation est-elle le fruit de l'activité d'un consommateur isolé ou d'un ensemble de consommateurs réunis en communauté (Cova, 2008 et 2012)?

\section{II - RÉSURGENCE DE L'AUTOPRODUCTION}

Cette confusion et ce trouble sur les notions de coproduction et cocréation sont à mettre en perspective avec la résurgence dans la littérature sur la consommation (Montéty, 2010) et sur le marketing (Troye et Supphellen, 2012) d'un concept,

Tableau 1 - Cocréation, coproduction et confusion

\begin{tabular}{|c|c|c|}
\hline $\begin{array}{l}\text { Conceptualisation } \\
\text { de l'activité } \\
\text { du consommateur }\end{array}$ & $\begin{array}{c}\text { Textes } \\
\text { de référence }\end{array}$ & $\begin{array}{l}\text { Incidences sur la description } \\
\text { de l'activité du consommateur }\end{array}$ \\
\hline $\begin{array}{l}\text { Similarité } \\
\text { de la cocréation et } \\
\text { de la coproduction }\end{array}$ & $\begin{array}{l}\text { Payne et al. (2008), } \\
\text { Xie et al. (2008) }\end{array}$ & $\begin{array}{l}\text { Les termes de cocréation, coproduction recouvrent } \\
\text { une même réalité } \\
\text { Ils peuvent donc être utilisés de manière équivalente } \\
\text { pour décrire l'ensemble des situations pour } \\
\text { lesquelles le consommateur joue un rôle actif dans } \\
\text { la production. }\end{array}$ \\
\hline Tout est cocréation & $\begin{array}{l}\text { Sheth et Uslay } \\
\text { (2007), } \\
\text { Vargo et Lusch } \\
\text { (2008) }\end{array}$ & $\begin{array}{l}\text { La coproduction n'est qu'un cas spécifique de } \\
\text { cocréation. } \\
\text { Il convient de bien connaître ce qu'est la cocréation } \\
\text { pour en délimiter les situations particulières relevant } \\
\text { de la coproduction. }\end{array}$ \\
\hline $\begin{array}{c}\text { La cocréation et } \\
\text { la coproduction sont } \\
\text { fondamentalement } \\
\text { différentes }\end{array}$ & Marion (2013) & $\begin{array}{l}\text { La cocréation consiste à faire advenir à l'existence } \\
\text { ce que l'on tire du néant : consommateur et } \\
\text { producteur participent simultanément à la création } \\
\text { d'une œuvre commune. La coproduction consiste à } \\
\text { exploiter des possibilités d'actions déterminées, en } \\
\text { vue d'un résultat attendu. } \\
\text { Il convient donc de bien dissocier ces deux termes. }\end{array}$ \\
\hline $\begin{array}{c}\text { La cocréation } \\
\text { n'existe que dans } \\
\text { l'interaction }\end{array}$ & Grönroos (2011) & $\begin{array}{l}\text { La cocréation n'a lieu que quand il y a coproduction. } \\
\text { On ne peut donc parler de cocréation sans } \\
\text { s'être préalablement assuré que producteurs et } \\
\text { consommateurs interagissent déjà autour de la } \\
\text { coproduction. }\end{array}$ \\
\hline
\end{tabular}


jusqu'alors relativement négligé, celui d'autoproduction. La place de l'autoproduction, fondement de la vie traditionnelle, a d'abord été réduite par l'industrialisation de la société (Chessel, 2012) puis par l'économie de marché qui a (re)distribué les rôles (Godbout, 2007) : d'un côté les producteurs qui produisent, de l'autre les consommateurs qui consomment et au milieu, les distributeurs agissant comme intermédiaires. L'autoproduction a ainsi été progressivement reléguée au rôle marginal de «production de biens ou de services en dehors de la production marchande " (Pinçon, 1986, p. 629). C'est cette vue restreinte de l'autoproduction qui a dominé dans le sens commun jusqu'il y a peu et qui l'a fait confondre avec l'autoconsommation. Cependant, l'individu occidental n'a jamais totalement adhéré à la division stricte des rôles et l'autoproduction en relation avec la production marchande a perduré tant sous la forme de détournement des produits et services offerts par le marché (Certeau, 1980) que de couplage de ceux-ci avec des ressources non marchandes comme dans le cas des « confitures, conserves familiales, tricots, petit mobilier, objets de décoration » (Certeau et Giard, 1983, p. 17). Cette activité productive du consommateur, loin de disparaître, est même plutôt en expansion dans nos sociétés postmodernes au travers des pratiques de bricolage, de personnalisation, de tuning, de loisirs créatifs et autres (Campbell, 2005). Selon le Credoc, de plus en plus d'individus mettent en place des stratégies d'autoproduction (Hébel, 2013). L'autoproduction est même perçue comme une alternative politique en opposition au monde d'ultraconsommation dans lequel baigne l'individu contemporain (Schor, 2010).
De fait, les pratiques d'autoproduction sont en pleine évolution (Montéty, 2010), ce qui explique la résurgence actuelle de ce concept. L'autoproduction n'est plus une activité - seulement - extérieure à la production marchande mais entremêlée avec celle-ci. De nombreux dispositifs marchands viennent s'encastrer dans les pratiques d'autoproduction comme le montre de Montéty (2010) au sujet de l'autoproduction croissante du pain par l'entremise d'une machine à pain : «La panification mécanisée à la machine est bien une autoproduction mais elle est "médiée" par un objet technique qui a une valeur propre, liée à sa forme et son statut » (Montéty, 2010, p. 103). Cette pratique ne doit pas être confondue avec l'activité de production au foyer pour le compte d'une entreprise, comme dans le cas des «putting out systems » (Lazerson, 1995), configurations économiques dans lesquelles les entreprises fournissent aux travailleurs ruraux à domicile des matières premières, voire des outils, avant de récupérer en échange d'un salaire le produit transformé. L'autoproduction est déclinée dans la littérature en deux catégories, que nous allons détailler, qui permettent de différencier deux formes de rôle actif du consommateur et, en conséquence, de rôle tenu par l'entreprise : l'autoproduction dite dirigée (Dujarier, 2008) et l'autoproduction dite accompagnée (Cerezuelle et Roustang, 2010).

\section{III - AUTOPRODUCTION DIRIGÉE ET AUTOPRODUCTION ACCOMPAGNÉE}

Depuis de nombreuses décennies, les entreprises de services requièrent une participation du consommateur (Eiglier 
et Langeard, 1987) qui prend la forme d'une autoproduction dirigée (Dujarier, 2008). À l'occasion de cette participation, le consommateur utilise un certain nombre de ressources propres (physiques, intellectuelles, affectives) qui se rajoutent et se combinent avec les ressources que l'entreprise mobilise dans son système d'offre. Ce processus est un système dynamique et varie selon le type et le degré des moyens et compétences mis en jeu. On observe aujourd'hui que cette autoproduction dirigée s'étend doublement: elle concerne un nombre croissant d'activités, d'une part, et les tâches productives réalisées par le consommateur sont de plus en plus nombreuses, d'autre part. Le phénomène dépasse ainsi largement les situations de service : il concerne aussi les produits à l'exemple du modèle Ikea. L'autoproduction du consommateur dirigée par l'entreprise consiste globalement en l'externalisation par l'entreprise de tâches simplifiées en partie automatisées et prises en charge par soi et pour soi par le consommateur (Dujarier, 2008). L'extension de la logique du self-service à de nombreuses situations incite ainsi le consommateur à produire en utilisant un support automatisé fourni par l'entreprise. Les exemples ne manquent pas: guichets électroniques d'impression et d'enregistrement de billets de train et d'avion, automates de vente, automates de service, self-scanning, hotlines en tous genres, caisses libre-service, assistances téléphoniques mais aussi modes d'emplois permettant de construire son armoire soimême (Troye et Supphellen, 2012), illustrent ce développement. Le consommateur d'aujourd'hui, lorsqu'il se sert d'un de ces supports, remplace une prestation autrefois fournie par le producteur : il doit réaliser la tâche prescrite (Dujarier, 2008) s'il veut accéder à la consommation. Les entreprises ne le motivent pas vraiment généralement, elles imposent cette organisation comme exprimée sur le site d'Ikea : «Les meubles Ikea sont conçus pour être assemblés facilement par le client. Cette formule demande un peu de temps et d'énergie, mais c'est la plus économique: vous ne payez pas pour le montage en usine!» $\mathrm{Si}$ on ne veut pas autoproduire, il faut s'adresser à un autre fournisseur. L'autoproduction dirigée nécessite un apprentissage à la fois technique et social, pour faire du consommateur un acteur compétent et productif. Celui qui ne suit pas ou qui n'arrive pas à faire face à ces systèmes de self-service est mis en difficulté pratique et morale, dans sa consommation quotidienne. De plus, si l'autoproduction dirigée du consommateur peut favoriser les contacts entre consommateurs dans certains cas (ils s'entraident et se forment), elle renforce globalement l'individualisation des activités.

L'autoproduction accompagnée a concerné, tout d'abord, les politiques volontaristes qui ont été menées pour rendre accessibles à tous les activités d'autoproduction (Cerezuelle et Roustang, 2010). L'autoproduction accompagnée a consisté à apporter une aide technique et sociale à des publics défavorisés pour leur permettre de faire par eux-mêmes. Elle se présente comme une démarche concrète d'accompagnement et de transmission des savoir-faire à travers des ateliers, des chantiers, etc. Depuis une vingtaine d'années, et à partir d'horizons très différents, des associations locales ont mis en place des actions d'accompagnement à l'autoproduction destinées à re(développer) les compétences des individus. Bien que l'autoproduction accompagnée ait été initiée 
par des organismes à vocation sociale, certaines entreprises utilisent maintenant cette approche dans un cadre dépassant celui de l'aide à un public défavorisé. De nombreuses initiatives d'ateliers dits de self-mécanique ont ainsi vu le jour ces toutes dernières années. Elles relèvent soit d'initiatives collectives telle celle de la FFMC (Fédération française des motards en colère), soit d'initiatives individuelles (Moto Self Services, Self Meca Auto, Self Mécanique, Self Auto Center, etc.). L'utilisation de l'anglais « self» traduit bien l'idée d'autoproduction induite dans ces initiatives. Dans les diverses offres lancées autour du garage en self-service avec accompagnement professionnel, les notions d'autonomisation et de passion sont prégnantes : les individus viennent apprendre à « mécaniquer tout seul » auprès d'un passionné d'automobile ou de moto qui fait et sait faire partager sa passion mais ne donne pas de cours. De même, Barilla avec son opération Casa Barilla, a ouvert des espaces éphémères dédiés aux plats de pâtes et à la cuisine italienne dans lesquels est proposée une école pour apprendre à préparer les pâtes : « Guidés par les prestigieux Chefs Academia Barilla, vous découvrirez comment réaliser les meilleures recettes de pâtes italiennes, aussi originales que savoureuses. À la fin du cours vous dégusterez les mets préparés autour d'une table conviviale et chaleureuse, en présence du chef Academia Barilla. » ${ }^{1}$

L'exemple d'autoproduction accompagnée souvent donné dans la littérature marketing est celui des magasins Games Workshop qui proposent le fameux jeu de guerre
Warhammer $^{2}$ : la tâche principale des vendeurs repose davantage sur l'initiation que la vente. Le personnel en contact joue ainsi le rôle de guide : « Le conseil et l'animation font partie intégrante des missions du vendeur. Il a un rôle pédagogique lorsqu'il apprend aux novices à peindre ou à jouer en indiquant les règles à mobiliser pour contrer telle ou telle attaque de l'armée adverse. " (Ezan et Cova, 2008, p. 54). Dans ce petit monde communautaire qu'est celui des passionnés de Warhammer, l'autoproduction de son armée par le consommateur est la règle : il doit peindre toutes ses figurines avant de pouvoir prétendre jouer et cette autoproduction est accompagnée par le personnel en contact de Games Workshop qui veille au développement de compétences du joueur. Au-delà des employés de Games Workshop, il peut compter sur l'entraide existant dans la communauté (Muniz et O’Guinn, 2001) pour apprendre à jouer : les experts aident les novices et tout le monde s'entraide au cours du jeu, car personne n'est capable de connaître l'ensemble des règles générales qui sont recensées dans un ouvrage de référence de 260 pages et qui sont ensuite déclinées dans les fameux Codex : trente livres de règles spécifiques dédiées à chaque armée, de plus de cent pages chacun.

\section{IV - VERS UN ÉLARGISSEMENT DES CATÉGORIES DE L'AUTOPRODUCTION}

Si l'autoproduction regroupe l'ensemble des pratiques dans lesquelles « les consom-

1. http://www.barilla.fr/casabarilla2012/index.html

2. Warhammer produit par Games Workshop est un jeu de stratégie militaire de plateau réalisé avec des figurines à peindre et à collectionner. 
mateurs jouent un rôle actif dans la création de produits finaux » (Troye et Supphellen, 2012, p. 33), une focalisation sur les seules activités d'autoproduction parait à même de corriger les effets de flou et de confusion générés par le zoom arrière inhérent au courant de la SDL et de la cocréation de valeur (Vargo et Lusch, 2011). Un zoom avant sur l'autoproduction du consommateur en relation avec l'entreprise permet d'abord de préciser deux grandes catégories de coproduction/cocréation en fonction de l'intention première du consommateur, même si son activité peut avoir des effets secondaires qui vont au-delà de cette intention et impactent l'autre catégorie : 1) les activités du consommateur ayant comme objectif premier la production/création pour soi et les siens, ce qui est recouvert par le terme d'autoproduction; 2) les activités du consommateur ayant comme objectif premier la production/création pour l'entreprise, ce qui se situe en dehors de l'autoproduction. Un zoom avant sur l'autoproduction permet aussi, comme nous l'avons vu, de préciser et différencier les pratiques des consommateurs actifs ainsi que le rôle de l'entreprise et d'autres acteurs potentiels en fonction de la catégorie d'autoproduction : dirigée ou accompagnée. Néanmoins, ces deux catégories ne permettent pas de rendre compte du caractère polymorphe et dynamique de l'autoproduction aujourd'hui : « Pour mieux saisir les dynamiques d'autoproduction actuelles, toutes les formes exemplaires méritent d'être étudiées pour leur propre compte pour ne pas trop vite assimiler leur essor à un "retour en arrière" et une rupture. » (Montéty, 2010, p. 103). Poursuivant dans la logique de zoom avant, nous proposons d'étudier des cas exemplaires d'autoproduction du consommateur en relation avec l'entreprise. Le but est double : détailler le jeu des acteurs de l'autoproduction et de leurs interactions pour chaque cas exemplaire et caractériser ces cas pour élargir la catégorisation de l'autoproduction.

La catégorisation est une opération d'abstraction consistant à classer diverses données résultant de l'observation selon un système de catégories, exhaustif ou non. La catégorisation est un processus inévitable dans le développement de la connaissance. La tradition aristotélicienne s'occupe ainsi de définir la liste des catégories, entendues comme les classes les plus fondamentales d'objets. Si la catégorisation est un processus incontestable, rien n'indique que les contenus et limites des catégories sont, eux aussi, incontestables (Baele, 2010). Au contraire, une catégorisation est toujours en évolution, ses catégories évoluant en fonction des résultats de la recherche. Dans le cas présent, la catégorisation de l'autoproduction n'a pas de vocation exhaustive et incontestable : elle représente le point actuel fait par les chercheurs à partir de leurs observations. Cette catégorisation pourra être enrichie par la suite à partir d'autres observations à même de faire émerger de nouvelles catégories d'autoproduction. Cette démarche s'appuie sur les catégorisations proposées dans la littérature (Carù et Cova, 2007 ; Cova, 2008) tout en précisant et (re)détaillant les catégories existantes. L'étude de cas exemplaires d'autoproduction relève d'une démarche empirique qualitative. Elle repose sur l'examen approfondi de deux cas dont les critères de sélection sont présentés par la suite. Pour chaque cas, les acteurs pensant, parlant et interagissant sont étudiés dans un contexte ou en situation (Flyjvberg, 2006). Enfin, 
cette catégorisation concerne les seules activités licites et autorisées par l'entreprise ; les contrefaçons et autres détournements interdits par l'entreprise sont volontairement écartés de notre champ d'étude pour des raisons éthiques.

La méthodologie retenue pour mener les investigations empiriques se fonde sur une étude ethnographique portant sur deux situations de consommation/production identifiées par les auteurs comme des cas inédits d'autoproduction. Le premier cas sélectionné concerne le concept Warhammer de Games Workshop qui a fait l'objet de plusieurs études empiriques qui ont mis au jour les principales occurrences susceptibles de définir une communauté de marque. Dans cet esprit, la porosité de la frontière entre vendeurs et consommateurs pour animer et promouvoir la marque a été relevée dans les contributions sur le sujet (Ezan et Cova, 2008). Les études précédentes ont également souligné le niveau d'engagement élevé des consommateurs qui conduit les passionnés de Warhammer à se dépasser pour produire des figurines les plus abouties possible en mobilisant les conseils et les astuces délivrés par Games Workshop. À la lumière des éclairages de l'autoproduction exposés précédemment, le cas Warhammer peut être qualifié en l'état d'autoproduction accompagnée. Néanmoins, les évolutions récentes du système d'offre relevées par les auteurs qui fréquentent régulièrement des fans de la marque et les points de vente dans lesquels ils exercent leur passion, conduisent à examiner ce cas à la lumière d'une nouvelle grille de lecture pour disposer d'une catégorisation prenant en compte les facettes diversifiées de l'autoproduction et rendre compte de son caractère dynamique. Le second cas Playmobil/Klikobil relève d'une situation d'autoproduction plus inédite dans la recherche académique. En effet, à notre connaissance, ce type de situation n'a pas bénéficié d'un éclairage conceptuel permettant de saisir la manière dont le consommateur détourne les produits d'une marque leader sur un marché pour en faire des productions personnelles en mobilisant les univers crées par l'entreprise Playmobil, ceci avec l'accord de l'entreprise. Ainsi, ce cas présente une originalité par rapport à l'existant dans la mesure où la situation présentée décrit un consommateur qui modifie le ciblage d'une offre initialement dédiée aux enfants pour en faire un concept innovant à destination des adultes. Par son travail, ce passionné manifeste son désir de s'affranchir de la tutelle de l'entreprise tout en conservant l'idéologie portée par les figurines Playmobil : variété des personnages, histoires à créer, diversité des métiers et des images convoquées (Hetzel, 2002). À ce titre, ce détournement accepté par Playmobil mérite d'être examiné afin d'enrichir les catégories de l'autoproduction.

La collecte des données repose sur une enquête de terrain permettant aux auteurs de disposer d'un corpus dense et varié (voir encadré ci-après). L'analyse de ce corpus fait ressortir deux situations d'autoproduction inédites que nous qualifions pour la première d'entre elles d'autoproduction facilitée et pour l'autre d'autoproduction émancipée.

\section{V - AUTOPRODUCTION FACILITÉE PAR L'ENTREPRISE}

L'étude du cas Warhammer montre un changement net par rapport à la situation étudiée par Ezan et Cova (2008). Au cours des cinq dernières années (2008-2013), il y a eu 


\section{RECUEIL ET ANALYSE DES DONNÉES}

L'approche ethnographique mobilisée pour examiner ces deux situations d'autoproduction se fonde sur une collecte de données diversifiées permettant de saisir de manière la plus fine possible le sens que les consommateurs donnent à leurs actions (Yin, 2009). Pour le cas Warhammer, l'approche combine des observations participantes menées par l'un des auteurs au sein de sa famille et dans les lieux de vente depuis 2003. Ces observations sont complétées par des entretiens semi-directifs, des photographies et des vidéographies réalisés lors des rassemblements organisés par l'entreprise ou par les consommateurs totalisant environ 200 clichés et 6 heures d'enregistrement. La démarche longitudinale mobilisée a permis de réactualiser le corpus obtenu en fonction de l'évolution du concept d'autoproduction détecté par les auteurs à partir de 2012. Cette réactualisation repose sur des observations participantes réalisées dans les points de vente tous les samedis après-midis durant deux mois. En ce qui concerne le cas Playmobil/Klikobil, l'approche est constituée d'observations participantes, menées par l'un des auteurs au sein de cette petite communauté pendant 12 mois. Ces observations sont enrichies par des entretiens phénoménologiques, des photographies et des vidéographies. Ce corpus se centre sur Olivier, fondateur de Klikobil, et a été collecté dans son atelier/magasin. Au total, le recueil de données comprend 7 heures d'enregistrements et 34 clichés. Ces données ont été analysées à partir d'une lecture flottante et rapprochées du cadre conceptuel de l'autoproduction pour en faire ressortir les éléments discriminants permettant de faire émerger deux nouvelles catégories.

évolution du rôle actif des consommateurs et parallèlement du rôle de l'entreprise qui ont fait s'éloigner l'ensemble d'un système d'autoproduction accompagnée comme vu précédemment pour développer une approche facilitant - sans accompagnement - l'autoproduction des joueurs de Warhammer. Nous constatons, en 2013, que le magasin a changé. Plus de rayonnages foisonnants laissant voir les boîtes de figurines, plus de figurines exposées sous blister, plus de vitrines montrant les nouvelles productions de la marque. L'espace est désormais occupé par des ateliers : peinture, montage, et surtout la fameuse table de jeu qui trône en bonne place au centre du magasin. Cette table occupe à présent une place plus importante qu'auparavant dans le magasin et permet ainsi à de nombreux fans de participer. Autour de la table, nous assistons à des scènes surprenantes où le personnel en contact est quasiment contraint de s'imposer pour jouer. Il en arrive dans certains cas, à demander la permission pour déplacer quelques figurines qui appartiennent au magasin. Manifestement, les fans se passeraient bien de ses services qui perturbent plutôt la partie. Celui-ci en effet, fait des remarques sur le déroulement de la partie qui n'intéressent manifestement personne car ces remarques dérangent la dynamique du groupe. Il alerte sur une règle à respecter devant le regard médusé voire outré des passionnés qui n'ont pas besoin qu'on leur rappelle cette règle qu'ils connaissent apparemment par cœur. Le vendeur se trouve 
alors en porte à faux car en indiquant une règle, il signifie qu'il n'a pas su évaluer le niveau d'expertise des joueurs et une pointe d'agacement se laisse voir parmi les passionnés. Celle-ci disparaît quand le vendeur est appelé pour encaisser un produit ce qui permet aux passionnés de se retrouver entre eux : "Il nous gonfle Seb avec ses conseils... » (Nicolas, 21 ans) ; "Ce serait sympa qu'il nous lâche, y a pas un client qui arrive... » (Julien, 23 ans) ; « Il va falloir qu'il renouvelle le lexique, on les connaît ses stratégies... » (Matthieu, 26 ans). De plus, les fans arrivent dans le magasin avec leur mallette remplie de figurines qu'ils ont montées, peintes et sculptées. Souvent ces autoproductions du consommateur sont bien plus professionnelles que celles délivrées par les vendeurs pour jouer dans le magasin: on sait ainsi que les figurines «moches» appartiennent au vendeur ou en l'occurrence au magasin et que les plus belles, les productions les plus abouties, ont été réalisées par les passionnés au prix de nombreuses heures de travail, d'enquête et de dextérité pour arriver à un tel niveau de finesse : « T'as vu la tronche de la figurine, ça peut pas être la mienne, tu me prends pour qui ?» (Damien, 17 ans) ; «J'apporte pas mes plus belles figurines, je ne veux pas leur faire honte. » (Thomas, 18 ans).

Pourquoi les consommateurs se rendent-ils en magasin pour jouer si comme auparavant ils ne souhaitent pas bénéficier de l'expertise, de la caution du vendeur assurant le bon déroulement d'une partie ? La réponse vient sans doute du fait de la maturité du concept qui fait que les passionnés sont arrivés eux-mêmes à un niveau d'expertise : ils peuvent se passer sans problème de la présence du personnel de contact; désormais c'est le magasin qui leur fournit un gage de reconnaissance de leur expertise. En d'autres termes, quand on vient aujourd'hui jouer à Warhammer chez Games Workshop c'est parce qu'on connaît bien les règles et qu'on veut se mesurer à d'autres passionnés qui souhaitent à leur tour rencontrer des joueurs à leur niveau en dehors de leur cercle d'amis. Cependant, et en conséquence de la situation à peine décrite autour de la table de jeu, les tables de peinture et de montage sont plus présentes qu'avant et accueillent un public plus nombreux pour pouvoir faire découvrir le concept et élargir la cible de consommateurs. Ici les vendeurs recrutés pour l'occasion sont très jeunes. Ils font figure non plus d'experts mais d'animateurs type « colos » ou « centres aérés », peignant avec les enfants, les aidant, les encourageant à ne pas renoncer en cas de difficulté et en montrant aux parents leur admiration pour un enfant doué. Ce qui tend encore à les marginaliser par rapport à ces passionnés qui jouent à côté. Ceci pose clairement le problème de la légitimité du personnel de contact. On a l'impression qu'il n'est plus à sa place dans le magasin et que la légitimation est désormais assumée par les passionnés qui font figure de " grands frères " pour ces enfants en quête de socialisation Warhammer.

Alors que la situation présentée dans les magasins Games Workshop autour des tables de peinture relève de l'autoproduction accompagnée, celle décrite autour de la table de jeu relève d'une autoproduction dans laquelle le personnel n'a plus de rôle actif. Son rôle comme celui de toute l'entreprise est de fournir une plateforme, le magasin et sa table de jeu, apte à faciliter l'autoproduction des consommateurs réu- 
nis en communauté qui n'ont besoin que de cela. Dans cette catégorie d'autoproduction en pleine expansion, l'entreprise fournit non pas un support directif comme dans le cas du self-service, mais plutôt une plateforme offline (magasin) ou online (site web) sur laquelle vont pouvoir s'agréger les consommateurs pour autoproduire leurs expériences. Sur ces plateformes, le consommateur n'interagit en général pas avec le personnel en contact mais plutôt avec d'autres consommateurs compétents et/ou passionnés comme lui. Le Bon Coin et eBay sont des cas notoires d'entreprises du net ayant mis en place les conditions de ce type d'autoproduction comme l'ont fait aussi les services de covoiturage, type BlaBlaCar (www.covoiturage.fr), qui équipent les entreprises, collectivités et événements souhaitant déployer un tel service pour leurs employés, clients ou visiteurs. Concernant eBay, Denegri-Knott et Zwick (2012) montrent, sur la base d'une étude qualitative de quarante utilisateurs, comment le système technique de sa plateforme incorpore un ensemble de règles et de paramètres qui, de fait, facilite l'autoproduction des consommateurs mais aussi limite leur autonomie. Il y a là une certaine prévisibilité des activités possibles qui rend l'autoproduction sur ce type de plateformes immatérielles moins excitante au fil du temps (Denegri-Knott et Zwick, 2012).

Globalement, l'autoproduction facilitée peut se définir comme une stratégie délibérée de l'entreprise visant à favoriser l'autonomie et donc la créativité des consommateurs réunis en communauté. Ici, le système d'offre est conçu pour encourager l'autoproduction des consommateurs tout en bordant assez précisément le périmètre des activités de cette autoproduction.

\section{VI - AUTOPRODUCTION ÉMANCIPÉE DE L'ENTREPRISE}

Le cas Playmobil/Klikobil renvoie à un lieu ouvert par Olivier avec deux amis en 2010 à Drémil-Lafage dans la banlieue toulousaine et dédié au rachat, à la restauration et la revente de vieux Playmobil. Le concept de ce lieu est unique en France voire même en Europe. Le magasin a une grande enseigne au nom de Klikobil mais de l'extérieur on voit clairement la référence à Playmobil et aux jouets de la marque allemande. Dans ce lieu, «l'univers merveilleux du petit bonhomme aux yeux ronds et au sourire légendaire » (dixit Olivier) est reconstitué en de nombreuses mises en scène. Ces mises en scène, régulièrement actualisées, sont réalisées par Olivier. Il imagine l'histoire ou le contexte et décide quelles figurines impliquer, comment les positionner les unes par rapport aux autres, de quel thème s'inspirer, quel récit raconter. Tout cela, bien sûr, est bien loin des scénarios tels qu'ils sont représentés dans les catalogues officiels Playmobil.

Dès son ouverture, Klikobil a eu maille à partir avec la PME allemande Playmobil. Alors que les trois associés, passionnés de Playmobil, pensaient ouvrir le lieu sous ce nom, l'entreprise les en a dissuadé. Ils ont alors choisi de nommer le lieu Klikobil en référence au nom d'origine des jouets Playmobil, Klicky, nom aujourd'hui abandonné par l'entreprise. Cette idée a été acceptée par l'entreprise allemande. Parmi les Klikobil réalisés par l'équipe, on découvre des personnages tels que Zorro, Tintin, ou encore Jean-Paul Gaultier. Dans l'atelier de Klikobil, les anciennes figurines Playmobil sont démontées : elles sont, en effet, composées de plusieurs éléments (chapeaux, vête- 
ments, chevaux, armures, manteau, etc.) et accessoires (armes, bâtons de fait, cheval, etc.). Olivier défait, sélectionne et recompose les pièces des vieux Playmobil que des parents ou des grands-parents ont retrouvés dans leur garage et sont venus échanger ou vendre. Olivier leur donne une nouvelle vie, et souvent une nouvelle identité, puis les remet en vente dans ses magasins ou dans sa boutique en ligne. Pour leur donner cette nouvelle vie, Olivier rassemble les pièces en prenant le chapeau d'une figurine, la capuche d'un autre, etc., pour créer, par exemple, un cheikh arabe, un toréador avec sa cape rouge, un chef cuisinier japonais avec son poisson à trancher, un juge avec son bureau, un cosaque, un ninja, etc. Dans ce lieu, les passionnés de Playmobil de tous âges viennent communier autour des réalisations d'Olivier. Le savoir-faire d'Olivier vient tout droit de son enfance, des heures passées à jouer avec des Playmobil, mais il s'agit aussi d'une compétence vivante et vitale qu'il fait partager à ses proches et à tous ses visiteurs. Dès qu'Olivier montre à un visiteur tel ou tel jouet, il commence à le faire vivre, à créer une histoire autour de la figurine, à lui donner un nom, et à la faire interagir avec d'autres jouets.

Avec Olivier et Klikobil, nous faisons face à un cas d'autoproduction des consommateurs qui s'inscrit encore dans une relation avec l'entreprise (ici Playmobil) tout en s'émancipant considérablement de ce lien. Cette autoproduction peut être qualifiée d'émancipée car, d'une part, le consommateur (ou plutôt «prosumer» dans le cas d'Olivier !) ne suit plus à la lettre les canons esthétiques de la marque mais les réinterprète sous de nouvelles formes et dans de nouvelles scènes (Muniz et Schau, 2007), et que, d'autre part, l'entreprise
Playmobil autorise tout en ne facilitant pas l'autoproduction des consommateurs (Berthon et al., 2007) ou, tout du moins, n'utilise pas de moyen juridique ou autre pour empêcher l'autoproduction des consommateurs sur la base de l'offre de l'entreprise. Pourtant, cela peut aller assez loin car cette autoproduction pour soi et pour les siens peut être étendue à tous les proches, c'est-à-dire à tous les membres d'une communauté qui partagent la même passion. C'est ce que Hein (2011) décrit dans le cas des fan-clubs de jeunes chanteurs et groupes qui produisent des objets à l'effigie de leur idole naissante car il n'en existe pas et qui, de fait, le font pour eux et pour leurs proches jusqu'à en vendre : «Je suis fan de Saez. À ses débuts, il n'avait aucun t-shirt à vendre lors de ses concerts. Du coup, j'ai dû faire mes propres t-shirts Saez. J'étais pas la seule d'ailleurs. » (Sonia, 23 ans, étudiante en M1) (Hein, 2011, p. 42).

L'autoproduction émancipée peut s'envisager comme un désir du consommateur de s'approprier les valeurs de la marque à partir de compétences spécifiques qu'il aura lui-même développées. Le système d'offre de l'entreprise lui sert alors de support pour mener ses propres aspirations créatives. Pour autant, il s'en détache car les produits de l'entreprise sont détournés (Aubert-Gamet, 1996) pour devenir des emblèmes identitaires du consommateur et de sa communauté. C'est sans doute la raison pour laquelle l'entreprise n'intervient pas dans ces mécanismes de transfert car l'autoproduction émancipée constitue un gage de reconnaissance des bénéfices de la marque, voire de la fascination qu'elle exerce auprès des passionnés, à l'image d'Ikea pour les Ikea hackers (http://www. ikeahackers.net). Bien que les consomma- 
teurs impliqués dans une autoproduction émancipée puissent cocréer de la valeur pour l'entreprise (Schau et al., 2009), ils ne le font pas en première intention. À l'instar des passionnés de Lego qui créent de nouveaux assemblages de briques et les exposent fièrement durant les fameuses « brickfairs » (http://www.brickfair.com/), ces consommateurs autoproduisent d'abord ces assemblages pour eux-mêmes et pour leurs proches. L'entreprise peut capitaliser sur leurs activités par une veille de ces autoproductions (Hatch et Schultz, 2010), mais ne peut pas les canaliser et orienter comme cela est fait dans les programmes collaboratifs. Cette autoproduction émancipée se positionne à l'extrême du continuum par rapport à l'autoproduction dirigée.

\section{VII - QUATRE CATÉGORIES D'AUTOPRODUCTION}

Le concept d'autoproduction permet un zoom avant sur les activités de cocréation $\mathrm{du}$ consommateur ayant comme objectif premier la production/création pour soi et les siens. Ce zoom met en lumière la tension existant entre le pouvoir du consommateur et celui de l'entreprise dans un cadre qui reste néanmoins licite et autorisé. Il met aussi en exergue la présence d'un troisième acteur qui vient souvent interférer dans le jeu: la communauté de consommateurs. Cette manière d'envisager la production du consommateur en confrontant le regard que l'entreprise porte à cette activité débouche sur quatre catégories d'autoproduction des consommateurs. Chaque catégorie correspond à un système spécifique de jeu d'acteurs qui se caractérise par des positions différentes sur les points suivants : rôle de l'entreprise, du consommateur et de la com- munauté. Le détail de ces trois positions permet d'examiner l'autoproduction sous un angle individuel et sous un angle collectif en montrant comment la communauté vient interférer dans la relation consommateur-entreprise (tableau 2).

Ainsi, si l'autoproduction se positionne tout le long d'un continuum en fonction du rôle respectif du consommateur, de la communauté et de l'entreprise, elle reste néanmoins dans un cadre circonscrit au sein duquel les trois acteurs-clés du système se nourrissent mutuellement du travail des autres soit par appropriation de compétences techniques, soit par captation d'éléments symboliques ou sociaux. Toutefois, les deux catégories que cette recherche a mises en lumière - l'autoproduction facilitée et l'autoproduction émancipée - rendent compte d'une porosité de plus en plus prégnante des activités de production dans les relations consommateur-communauté-entreprise et de la manière dont le consommateur expert et passionné déplace l'entreprise aux confins de ces activités.

Le continuum allant de l'autoproduction dirigée à l'autoproduction émancipée met en avant une baisse progressive du contrôle de l'entreprise sur les activités et une augmentation des compétences des consommateurs comme suit :

1) Forcer les consommateurs à faire une activité au moyen de dispositifs type automate, kit, etc. : les activités à effectuer sont simples et calibrées, le niveau de compétences requis n'est pas élevé ;

2) Guider les consommateurs dans certaines activités au moyen d'une plateforme et/ou du personnel en contact : les activités à effectuer sont complexes mais relativement calibrées, le niveau de compétences requiert un apprentissage ; 
Tableau 2 - Catégorisation de l'autoproduction et rôle des acteurs concernés

\begin{tabular}{|c|c|c|c|c|}
\hline $\begin{array}{c}\text { Catégorie } \\
\text { d'auto- } \\
\text { production }\end{array}$ & $\begin{array}{l}\text { Dirigée par } \\
\text { l'entreprise }\end{array}$ & $\begin{array}{l}\text { Accompagnée } \\
\text { par l'entreprise }\end{array}$ & $\begin{array}{l}\text { Facilitée par } \\
\text { l'entreprise }\end{array}$ & $\begin{array}{l}\text { Émancipée de } \\
\text { l'entreprise }\end{array}$ \\
\hline Exemples & $\begin{array}{l}\text { Ikea } \\
\text { RATP }\end{array}$ & $\begin{array}{l}\text { Self garage } \\
\text { Casa Barilla }\end{array}$ & $\begin{array}{l}\text { Bla-Bla Car } \\
\text { eBay } \\
\text { Warhammer }\end{array}$ & $\begin{array}{c}\text { Fan-club } \\
\text { Lego Brickfairs } \\
\text { Klikobil }\end{array}$ \\
\hline $\begin{array}{l}\text { Rôle majeur } \\
\text { de l'entreprise } \\
\text { (et objectif } \\
\text { latent) }\end{array}$ & $\begin{array}{c}\text { Forcer les } \\
\text { consommateurs } \\
\text { à faire une } \\
\text { activité. } \\
\text { (Modifier les } \\
\text { termes de la } \\
\text { transaction pour } \\
\text { augmenter la } \\
\text { rentabilité) }\end{array}$ & $\begin{array}{c}\text { Guider les } \\
\text { consommateurs } \\
\text { dans certaines } \\
\text { activités. } \\
\text { (Bâtir une } \\
\text { relation } \\
\text { privilégiée } \\
\text { avec les } \\
\text { consommateurs) }\end{array}$ & $\begin{array}{c}\text { Délimiter } \\
\text { un ensemble } \\
\text { d'activités } \\
\text { à effectuer } \\
\text { de manière } \\
\text { autonome par les } \\
\text { consommateurs. } \\
\text { (Valoriser la } \\
\text { créativité des } \\
\text { consommateurs) }\end{array}$ & $\begin{array}{c}\text { Autoriser } \\
\text { un ensemble } \\
\text { d'activités } \\
\text { effectuées } \\
\text { de manière } \\
\text { indépendante } \\
\text { par les } \\
\text { consommateurs. } \\
\text { (Circonscrire la } \\
\text { créativité des } \\
\text { consommateurs) }\end{array}$ \\
\hline $\begin{array}{l}\text { Rôle majeur du } \\
\text { consommateur } \\
\text { (et objectif } \\
\text { latent) }\end{array}$ & $\begin{array}{l}\text { Effectuer des } \\
\text { activités simples } \\
\text { et calibrées. } \\
\text { (Avoir accès à la } \\
\text { consommation) }\end{array}$ & $\begin{array}{l}\text { Apprendre } \\
\text { à effectuer } \\
\text { des activités } \\
\text { complexes } \\
\text { calibrées ou non. } \\
\text { (Développer des } \\
\text { compétences } \\
\text { dans une logique } \\
\text { fonctionnelle) }\end{array}$ & $\begin{array}{c}\text { Effectuer/ } \\
\text { organiser } \\
\text { de manière } \\
\text { autonome des } \\
\text { activités. } \\
\text { (Développer des } \\
\text { compétences } \\
\text { dans une logique } \\
\text { identitaire) }\end{array}$ & $\begin{array}{c}\text { Effectuer/ } \\
\text { organiser } \\
\text { de manière } \\
\text { indépendante des } \\
\text { activités. } \\
\text { (Auto-organiser } \\
\text { son activité tout } \\
\text { en maintenant } \\
\text { la relation avec } \\
\text { l'entreprise) }\end{array}$ \\
\hline $\begin{array}{l}\text { Rôle majeur de } \\
\text { la communauté } \\
\text { (et objectif } \\
\text { latent) }\end{array}$ & $\begin{array}{c}\text { Assister les } \\
\text { consommateurs } \\
\text { incapables } \\
\text { d'effectuer les } \\
\text { tâches requises. } \\
\text { (Proposer aide } \\
\text { et conseil) }\end{array}$ & $\begin{array}{c}\text { Fournir } \\
\text { des guides } \\
\text { auxiliaires } \\
\text { pour accroître } \\
\text { l'autonomie des } \\
\text { consommateurs. } \\
\text { (Développer } \\
\text { l'entraide) }\end{array}$ & $\begin{array}{c}\text { Fournir les } \\
\text { partenaires/ } \\
\text { ressources } \\
\text { nécessaires } \\
\text { à l'activité } \\
\text { autonome. } \\
\text { (Partager les } \\
\text { compétences) }\end{array}$ & $\begin{array}{c}\text { Fournir les } \\
\text { partenaires/ } \\
\text { ressources } \\
\text { nécessaires } \\
\text { à l'activité } \\
\text { indépendante. } \\
\text { (Promouvoir } \\
\text { l'expertise) }\end{array}$ \\
\hline
\end{tabular}

3) Délimiter un ensemble d'activités à effectuer de manière autonome par les consommateurs sur une plateforme: les activités à effectuer sont simples et bien délimitées, le niveau de compétences nécessaire est acquis ;
4) Autoriser un ensemble d'activités effectuées de manière indépendante par les consommateurs: les activités effectuées sont complexes et non calibrées ; le niveau de compétences est élevé. 
Une entreprise peut permettre aux consommateurs d'interagir selon un ou plusieurs modes d'autoproduction. Elle peut user l'autoproduction dirigée avec une grande partie de sa clientèle à l'image d'Ikea ou de Lego tout en autorisant et même promouvant une autoproduction émancipée à l'image des Ikea hackers ou des Lego brickfairs avec une partie restreinte des consommateurs réunis en communauté. Les quatre catégories ne correspondent pas à des positions figées mais s'insèrent dans un continuum qui évolue en fonction de jeux d'acteurs consistant à imposer son choix à l'autre protagoniste. Pour l'entreprise, ces choix s'opèrent a priori quand il s'agit de délimiter les marges de manœuvre accordées aux consommateurs dans la chaîne de valeur et a posteriori quand elle doit naviguer entre laisser-faire et contrôle pour recadrer l'activité du consommateur dans les limites qu'elle s'est fixée. Du côté du consommateur, ces décisions prennent corps dans son désir plus ou moins affirmé de se conformer au statut que l'entreprise a prévu pour lui ou de s'en affranchir en diffusant des productions parallèles à forte valeur ajoutée. Concernant la communauté, ces choix dessinent une place de protagoniste d'autant plus importante que l'entreprise lui transfère une partie croissante des responsabilités de fonctionnement.

Cette catégorisation de l'autoproduction laisse entrevoir le périmètre d'actions au sein duquel l'entreprise est amenée à naviguer pour contrôler l'activité du consommateur. Ces actions oscillent entre stratégie délibérée (autoproduction dirigée - autoproduction accompagnée) et stratégie émergente (autoproduction facilitée - autoproduction émancipée). L'autoproduction apparaît par conséquent comme la résul- tante des arbitrages menés par l'entreprise sur trois volets principaux :

- Nature de la relation nouée avec le consommateur: cette relation se conçoit de manière individuelle ou de manière collective. Ce choix n'est pas anodin dans la mesure où il invite le consommateur à une appropriation de l'offre seul ou assisté par rapprochement à une communauté de consommateurs. Or l'inscription de cette appropriation dans un registre collectif conduit le consommateur à se dépasser pour être reconnu dans sa communauté, ce qui favorise des logiques d'autoproduction facilitée ou émancipée ;

- Dimension temporelle de la relation: l'activité du consommateur s'inscrit dans un espace-temps. Plus le temps dédié à la consommation d'une offre s'éloigne d'une logique purement transactionnelle pour $s$ 'inscrire dans une relation durable alimentée par les innovations de l'entreprise en termes de produits ou de services, plus cette offre devient inspirante pour la créativité du consommateur ; ce qui contribue à une activité d'autoproduction conduite en parallèle de l'entreprise ;

- Bénéfices apportés par la relation: la création de valeur est ancrée soit sur des bénéfices utilitaires (gain de temps, économie financière, etc.), soit sur des bénéfices sociaux (intégration d'une communauté, renforcement identitaire fondé sur une passion, etc.) ; on peut ainsi concevoir que les bénéfices sociaux associés à un système d'offre sont de nature à conduire le consommateur à développer une solide expertise l'invitant peu à peu à s'affranchir du cadre prescrit par l'entreprise.

Concrètement, la catégorisation proposée fournit une grille de lecture permettant à l'entreprise de réfléchir à la conception de 
son système d'offre et aux signaux qu'elle envoie pour orienter l'activité du consommateur; ces signaux traduisent la nature du contrôle a priori ou a posteriori qu'elle peut exercer sur cette activité.

\section{CONCLUSION}

L'autoproduction est loin d'être aujourd'hui une simple production de biens ou de services en dehors de la production marchande comme son sens traditionnel le laisse encore supposer. Une focalisation sur le phénomène actuel d'autoproduction du consommateur en relation avec l'entreprise permet de disposer d'une vue systémique du jeu de trois acteurs principaux et de leurs interactions : entreprise - consommateur - communauté. En ceci, l'autoproduction suggère une articulation de ces interactions en montrant que l'activité du consommateur s'opère toujours aux confins des éléments techniques ou symboliques que l'entreprise véhicule et que la communauté transforme pour lui donner du sens. Sur le plan théorique, cette recherche ordonne la connaissance concernant une partie bien délimitée du phénomène de brouillage des frontières entre les mondes de la consommation et de la production. Elle évite en cela d'augmenter le flou lié à l'usage des notions englobantes de cocréation et de coproduction. Sur le plan méthodologique, ce travail confirme l'intérêt d'une immersion de longue durée sur un terrain pour rendre compte de l'évolution des interactions consommateur-entreprise et envisager ces interactions dans un continuum. Enfin, sur le plan managérial, cette recherche fournit une grille de lecture permettant aux entreprises d'identifier les leviers à activer tout au long du continuum de l'autoproduction et prendre des décisions appropriées en fonction de la stratégie qu'elles souhaitent adopter. Elle invite également à réfléchir aux limites de l'autoproduction et à la nature de l'appropriation du service souhaitée par le client. En d'autres termes, l'entreprise doit intégrer que, in fine, c'est le consommateur qui décide de la forme d'autoproduction qui lui convient le mieux.

\section{BiBLIOGRAPHIE}

Arnould E.J. (2007). "Consuming experience, retrospects and prospects". Consuming Experience, Carù A. et Cova B., Eds, Oxford, Routledge, p. 185-194.

Arnould E.J. et Thompson C.J. (2005). "Consumer Culture Theory (CCT): Twenty years of research", Journal of Consumer Research, vol. 31, n 4, p. 868-882.

Aubert-Gamet V. (1996). Le design d'environnement commercial dans les services: Appropriation et détournement par le client, Thèse de doctorat en gestion Université AixMarseille III.

Baele S. (2010). "Une approche multiple de la catégorisation pour projet éthique », Émulations, URL : http://www.revue-emulations.net/archives/n8/edito8

Berthon P.R., Pitt L.F., McCarthy I. et Kates S.M. (2007). "When customers get clever: Managerial approaches to dealing with creative consumers", Business Horizons, vol. 50, p. 39-47. 
Bonnemaizon A., Cadenat S., Benoit-Moreau F. et Renaudin V. (2012). « Consommateur "exécutant", "assistant marketing opérationnel", "relais" ou "apporteur de solutions": Dis-moi ce que tu fais, je te dirai qui tu es ! », Management \& Avenir, n 52, p. 175-193.

Campbell C. (2005). "The craft consumer culture. Craft and consumption in a postmodern society", Journal of Consumer Culture, vol. 5, n 1, p. 23-42.

Carù A. et Cova B. (Eds.) (2007). Consuming Experiences, Oxon, Routledge.

Cérézuelle D. et Roustang G. (2010). L'autoproduction accompagnée: un levier de changement, Toulouse, Eres.

Certeau M. (de) (1980). L'invention du quotidien : Arts de faire, Paris, Gallimard.

Certeau M. (de) et Giard L. (1983). «L'ordinaire de la communication », Réseaux, vol. 1, $\mathrm{n}^{\circ} 3$, p. 3-26.

Chessel M.E. (2012). Histoire de la consommation, Paris, La Découverte.

Cova B. (2008). "Consumer Made: Quand le consommateur devient producteur», Décisions Marketing, avril-juin, $\mathrm{n}^{\circ}$ 50, p. 19-27.

Cova B. (2012). "La mise au travail des consommateurs passionnés: le cas de la collaboration entre Alfa Romeo et les Alfistes », Sciences de la Société, n 82, p. 81-103.

Denegri-Knott J. et Zwick D. (2012). "Tracking prosumption work on eBay: Reproduction of desire and the challenge of slow re-McDonaldization", American Behavioral Scientist, avril, vol. 56, p. 379-398.

Dujarier M.A. (2008). Le travail du consommateur, Paris, La Découverte.

Eiglier P. et Langeard E. (1987). Servuction. Le marketing des services, Paris, McGraw-Hill.

Epp A.M. et Price L.L. (2011). "Designing solutions around customer network identity goals", Journal of Marketing, mars, vol. 75, p. 36-54.

Ezan P. et Cova B. (2008). « La confusion des rôles de consommateurs et de producteur dans les communautés de marques : une complicité dangereuse ? », Décisions Marketing, octobre-décembre, $\mathrm{n}^{\circ}$ 52, p. 51-60.

Flyjvberg B. (2006), "Five misunderstandings about case-study research", Qualitative Inquiry, avril, vol. 12, $\mathrm{n}^{\circ}$ 2, p. 219-245.

Godbout J.T. (2007). Ce qui circule entre nous. Donner, recevoir, rendre, Paris, Seuil.

Grönroos C. (2011). "Value co-creation in service logic: A critical analysis", Marketing Theory, vol. 11, n 3, p. 279-301.

Hatch M.J. et Schultz M. (2010). "Toward a theory of brand co-creation with implications for brand governance", Journal of Brand Management, vol. 17, n 8, p. 590-604.

Hébel P. (2013). La révolte des moutons - Les consommateurs au pouvoir, Paris, Autrement. Hein F. (2011). «Le fan comme travailleur: les activités méconnues d'un coproducteur dévoué », Sociologie du travail, vol. 53, p. 37-51.

Hetzel P. (2002). Planète Conso, Marketing expérientiel et nouveaux territoires de consommation, Paris, Éditions d'Organisation.

Humphreys A. et Grayson K. (2008). "The intersecting roles of consumer and producer: a critical perspective on co-production, co-creation and prosumption", Sociology Compass, vol. $2, \mathrm{n}^{\circ} 3$, p. 963-980. 
Lazerson M. (1995). “A New Phoenix? Modern Putting-out in the Modena Knitwear Industry", Administrative Science Quarterly, vol. 40, n 1, p. 34-59.

Maffesoli M. (2000). "Trouver les mots », Préface à la $3^{\text {e }}$ édition de l'ouvrage Le temps des tribus. Le déclin de l'individualisme dans les sociétés postmodernes, Paris, La Table Ronde.

Marion G. (2013). « La formation de la valeur pour le client : interactions, incertitudes et cadrages », Perspectives Culturelles de la Consommation, $\mathrm{n}^{\circ} 3$, à paraître.

Montéty C. (de) (2010). "La société de consommation dans le pétrin », Sociologies Pratiques, n $^{\circ}$ 20, p. 97-106.

Moss Kanter K. (2011). "Managing yourself: zoom in, zoom out", Harvard Business Review, February, p. 112-116.

Muniz A.M. Jr. and O'Guinn T.C. (2001). "Brand community", Journal of Consumer Research, mars, vol. 27, p. 412-432.

Muniz A.M. Jr. et Schau H.J. (2007). "Vigilante marketing and consumer-created communications", Journal of Advertising, vol. 36, n 3, p. 187-202.

Payne A.F., Storbacka K. et Frow P. (2008). "Managing the co-creation of value", Journal of the Academy of Marketing Science, vol. 36, $\mathrm{n}^{\circ}$ 1, p. 83-96.

Pinçon M. (1986). «Autoproduction, sociabilité et identité dans une petite ville ouvrière », Revue Francaise de Sociologie, vol. 27, n 4, p. 629-653.

Ritzer G. et Jurgenson N. (2010). "Production, consumption, prosumption: the nature of capitalism in the age of the digital "prosumer"', Journal of Consumer Culture, vol. 10, $\mathrm{n}^{\circ} 1, \mathrm{p} .13-36$.

Schau H.J., Muniz A.M. et Arnould E.J. (2009). "How brand community practices create value", Journal of Marketing, vol. 73, n 5, p. 30-51.

Schor J.B. (2010). Plenitude: the new economics of true wealth, New York, The Penguin Press.

Sheth J. et Islay C. (2007). "Implications of the revised definition of marketing: From exchange to value creation", Journal of Public Policy \& Marketing, vol. 26, n² 2, p. 302-307.

Troye S.V. et Supphellen M. (2012). "Consumer participation in coproduction: 'I made It Myself' effects on consumers' sensory perceptions and evaluations of outcome and input product", Journal of Marketing, mars, vol. 76, p. 33-46.

Vargo S.L. et Lusch R.F. (2004). "Evolving to a new dominant logic for marketing”, Journal of Marketing, vol. 68, $\mathrm{n}^{\circ} 1$, p. 1-18.

Vargo S.L. et Lusch R.F. (2008). "Service-dominant logic: continuing the evolution", Journal of the Academy of Marketing Science, vol. 36, n 1, p. 1-10.

Vargo S.L. et Lusch R.F. (2011). "It's all B2B... and beyond: Toward a systems perspective of the market", Industrial Marketing Management, vol. 40, n 2, p. 181-187.

Xie C., Bagozzi R.P. et Troye S.V. (2008). "Trying to prosume: toward a theory of consumers as co-creators of value", Journal of the Academy of Marketing Science, vol. 36, $\mathrm{n}^{\circ} 1$, p. 109-122.

Yin R. (2009). Case Study Research: Design and Methods, 4édition, London, Sage. 
